\title{
Weather, climate control, and imipenem-resistance in Acinetobacter baumannii: an ecological approach
}

\author{
Mônica da Silveira PhD ${ }^{1,2}$, Lucas Porangaba da Silva $\mathrm{MSc}^{1}$, Zildene Pedrosa de Oliveira Emídio PhD ${ }^{3}$ and \\ Carlos Magno Castelo Branco Fortaleza MD, PhD ${ }^{2}$ \\ ${ }^{1}$ Bauru State Hospital, São Paulo State Health Department, City of Bauru, São Paulo State Brazil, ${ }^{2}$ São Paulo State University (UNESP), Botucatu School of \\ Medicine, City of Botucatu, São Paulo State, Brazil and ${ }^{3}$ São Paulo State University (UNESP), Institute of Meteorological Research (IPMet), City of Bauru, São \\ Paulo State, Brazil
}

To the Editor-The incidence of healthcare-associated infections (HCAIs) caused by gram-negative bacilli (GNB) increases in summer ${ }^{1}$ and has been associated to outside environmental temperature $e^{2}$ and low latitudes. ${ }^{3}$ Evidence has been consistent for temperate $e^{4}$ and, to a lesser extent, tropical climates. ${ }^{5}$ The factors underlying this phenomenon and implications for infection control are not clear.

One of the causal hypotheses proposes that environmental reservoirs outside healthcare settings increase in warmer periods and that microorganisms are somehow carried into hospitals. ${ }^{4,6}$ This hypothesis is based on the following findings: (1) seasonality and meteorological dependence occur even in hospitals with complete climate control ${ }^{4}$; (2) polyclonal increase of Acinetobacter baumannii during summer, suggesting sources other than cross transmission; ${ }^{7}$ (3) seasonality for overall but not for multidrug-resistant $A$. baumannii, implying a possible communityassociated source. $^{8}$

We designed an ecological study aimed at testing premises that are central to the hypothesis that there are differences in seasonal pattern and influence of weather on the incidence of $A$. baumannii HCAIs according to climatization of hospital units or to imipenem resistance. The study was conducted in a teaching hospital in inner Brazil (Hospital Estadual Bauru, 335 beds). In that hospital, intensive care units (ICUs) have climate control, but all the other hospital wards are not climatized.

We obtained data from records of patient with cultures positive for A. baumannii from 2006 through 2017. Clinical cultures collected after the day 3 of admission were included (ie, the 3-midnight rule). ${ }^{9}$ We included only the first culture positive for A. baumannii for each subject. Monthly incidence was calculated for overall A. baumannii and for subgroups based on unit of admission, specimen, and resistance to imipenem.

Monthly meteorological parameters (ie, average temperature, average relative humidity, and aggregated rainfall) were collected from a nearby meteorological station (Institute of Meteorological Research, State University of São Paulo, City of Bauru, São Paulo State, Brazil).

Author for correspondence: Carlos Magno Castelo Branco Fortaleza, Departamento de Doenças Tropicais, Faculdade de Medicina de Botucatu, Distrito de Rubião Júnior, S/N ZIP/CEP 18618-970 Botucatu, São Paulo State, Brazil. E-mail: cmfortaleza@uol.com.br

Cite this article: da Silveira M, et al. (2018). Weather, climate control, and imipenemresistance in Acinetobacter baumannii: an ecological approach. Infection Control \& Hospital Epidemiology 2018, 39, 1133-1135. doi: 10.1017/ice.2018.171
We tested time series for seasonality using Box-Jenkins models and autocorrelation plots. ${ }^{10}$ The association with weather was tested using Poisson regression. Meteorological parameters were dichotomized, based on the 75th percentile of monthly values (temperature, $24.7^{\circ} \mathrm{C}\left[76.5^{\circ} \mathrm{F}\right]$; relative humidity, $79.6 \%$; rainfall, $163.6 \mathrm{~mm})$. Analyses were performed using NCSS 9 software (LLC, Kaysville, UT) and SPSS version 20 software (IBM, Armonk, NY).

We identified 1,207 patients with HCAIs due to A. baumannii. The overall incidence was 12.99 per 10,000 patient days: 67.12 for ICUs and 5.11 for noncritical wards. The imipenem resistance rate was $78.9 \%$.

Time series of incidence rates (either of overall A. baumannii or of subgroups) tested negative for seasonality. The results of our analysis of association with weather are presented in Table 1. We found a significant positive association with temperature for overall A. baumannii. The analyses of subgroups identified stronger associations with temperature for ICUs, isolates from blood cultures, and those that were imipenem resistant.

The absence of seasonality in Box-Jenkins models may be due to the poorly defined pattern of seasons in the tropical climate. The area where the study hospital is located has a mostly warm and humid period (usually October through March, including summer and spring), with lower temperatures in the rest of the year. However, this pattern is irregular, and brief increases in environmental temperature often occur, even during fall and winter.

A different picture emerges when we consider associations of outside temperature and the incidence of A. baumannii. This association was stronger in the following conditions: positive blood cultures, ICUs, and imipenem-resistant strains. The recovery of $A$. baumannii from blood cultures may be interpreted either as a sign of "true infection" (as opposed to colonization) or as a marker of the invasiveness of strains. Either way, our findings reinforce the impact of temperature on the incidence of HCAIs caused by this agent.

While the severity of critical patients is an obvious risk factor for acquisition of $A$. baumannii, the causes underlying the impact of the outside temperature on its incidence in ICUs are not straightforward. We are even less certain why we found greater association of temperature with imipenem-resistant strains. Notably, this finding is contrary to the picture described by 
Table 1. Poisson Regression Model for Impact of Monthly Meteorological Parameters on the Incidence of Healthcare-Associated Infections Caused by Acinetobacter baumannii

\begin{tabular}{|c|c|c|c|c|}
\hline \multirow[b]{2}{*}{ Culture Site/Unit of Admission } & \multirow[b]{2}{*}{ Incidence ${ }^{a}$} & \multicolumn{3}{|c|}{$\operatorname{RR}(95 \% \mathrm{Cl})^{\mathrm{b}}$} \\
\hline & & Temperature & Humidity & Rainfall \\
\hline Total clinical cultures & 12.99 & $1.09(1.02-1.16)$ & $0.97(0.91-1.05)$ & $0.93(0.77-1.01)$ \\
\hline Clinical cultures (ICUs) & 67.12 & $1.06(0.98-1.15)$ & $1.04(0.95-1.14)$ & $0.95(0.87-1.04)$ \\
\hline Blood cultures & 2.43 & $1.26(1.09-1.46)$ & $0.89(0.74-1.06)$ & $0.87(0.73-1.05)$ \\
\hline Blood cultures (ICUs) & 14.05 & $1.23(1.04-1.46)$ & $1.03(0.84-1.26)$ & $0.88(0.71-1.08)$ \\
\hline Blood cultures (noncritical wards) & 0.74 & $1.27(0.96-1.69)$ & $0.53(0.33-0.86)$ & $0.88(0.61-1.28)$ \\
\hline \multicolumn{5}{|l|}{ Imipenem-resistant $A$. baumannii } \\
\hline Clinical Cultures (noncritical wards) & 3.57 & $1.20(1.06-1.37)$ & $0.90(0.77-1.05)$ & $0.89(0.76-1.04)$ \\
\hline Blood cultures & 1.82 & $1.38(1.17-1.62)$ & $0.94(0.77-1.15)$ & $0.86(0.70-1.06)$ \\
\hline Blood cultures (ICUs) & 11.09 & $1.34(1.11-1.60)$ & $1.07(0.85-1.33)$ & $0.83(0.65-1.06)$ \\
\hline Boold cultures (noncritical wards) & 0.47 & $1.43(1.03-2.02)$ & $0.62(0.37-1.04)$ & $1.01(0.66-1.55)$ \\
\hline
\end{tabular}

Note. RR, rate ratio; $\mathrm{Cl}$, confidence interval; ICUs, intensive care units.

ancidence in cases per 10,000 patient days.

${ }^{\mathrm{b}}$ Meteorological values were dichotomized at the $75^{\text {th }}$ percentile of monthly values (temperature, $24.7^{\circ} \mathrm{C}\left[76.5^{\circ} \mathrm{F}\right]$; relative humidity, $79.6 \%$; rainfall, $163.6 \mathrm{~mm}$ ). Statistically significant associations $(P<.05)$ are presented in boldface.

Fukuta et al. ${ }^{8}$ Because infections caused by imipenem-resistant isolates (which are most likely healthcare-associated) increase in warmer months, we cannot infer that reservoirs in the outside environment are implicated in this phenomenon. On the other hand, increases in incidence according to outside temperature, even among patients admitted to units with complete climate control, are unlikely to be due to inanimate reservoirs within those units. Finally, because greater incidence of the pathogen of interest was not associated with any particular month or season, we can rule out the hypothesis that understaffing due to summer vacations would lead to greater transmission of nosocomial pathogens. ${ }^{4,6}$

We should approach our findings in terms of implications for research and implications for practice. Studies focusing on changes in healthcare work processes (eg, adherence to hand hygiene, conformities in isolation precautions) in different periods of the year or under different weather conditions should be conducted. Also, research including molecular strain typing could help determine whether there is increased cross transmission of A. baumannii (as well as other GNB) in periods of higher outside temperature. The obvious implication for practice is the requirement of intensifying infection control measures during warm months. Other recommendations may arise from continuing research.

The phenomenon of seasonality and meteorological determination of HCAIs caused by GNB remains puzzling. Still, from our perspective, it should not be regarded as mere curiosity. Instead, its elucidation may provide novel opportunities for infection prevention and control.

\section{Acknowledgments}

Financial support. C.M.C.B.F. received a research grant from the Brazilian National Council for Scientific and Technological Development (CNPq, process 312149/2015-8). The sponsor was not involved in the conduct of the study nor in the preparation, submission, and review of the manuscript.

Conflicts of interest. All authors report no conflicts of interest relevant to this article.

\section{References}

1. Perencevich EN, McGregor JC, Shardell M, et al. Summer peaks in the incidences of gram-negative bacterial infection among hospitalized patients. Infect Control Hosp Epidemiol 2008;29:1124-1131.

2. Schwab F, Gastmeier P, Meyer E. The warmer the weather, the more gram-negative bacteria-impact of temperature on clinical isolates in intensive care units. PLoS One 2014;9:e91105.

3. Fisman D, Patrozou E, Carmeli Y, et al. Geographical variability in the likelihood of bloodstream infections due to gram-negative bacteria: correlation with proximity to the equator and health care expenditure. PLoS One 2014;9:e114548.

4. Richet H. Seasonality in gram-negative and healthcare-associated infections. Clin Microbiol Infect 2012;18:934-940.

5. Fortaleza CM, Caldeira SM, Moreira RG, et al. Tropical healthcare epidemiology: weather determinants of the etiology of bloodstream infections in a Brazilian hospital. Infect Control Hosp Epidemiol 2014;35:85-88.

6. Eber MR, Shardell M, Schweizer ML, Laxminarayan R, Perencevich EN. Seasonal and temperature-associated increases in gram-negative bacterial bloodstream infections among hospitalized patients. PLoS One 2011;6: e25298. 
7. Christie C, Mazon D, Hierholzer W Jr, Patterson JE. Molecular heterogeneity of Acinetobacter baumannii isolates during seasonal increase in prevalence. Infect Control Hosp Epidemiol 1995;16:590-594.

8. Fukuta Y, Clarke LG, Shields RK, Wagener MM, Pasculle AW, Doi Y. Lack of seasonality in the occurrence of multidrug-resistant Acinectobacter baumannii complex. Infect Control Hosp Epidemiol 2012;33:1051-1052.
9. Cohen AL, Calfee D, Fridkin SK, et al. Recommendations for metrics for multidrug-resistant organisms in healthcare settings: SHEA/ HICPAC position paper. Infect Control Hosp Epidemiol 2008;29: 901-913.

10. Box GE, Jenkins GM, Reinsel CG. Time series analysis: forecasting and control. Hoboken NJ: Wiley; 2015.

\title{
Dissemination of Acinetobacter baumannii OXA-23 in old and new intensive care units without transfer of colonized patients
}

\author{
Rafael Renato Brondani Moreira MSc, Giselle Fukita Viana PhD, Amanda Carina Coelho de Moraes MD, \\ Marina de Souza Bastos PhD, Sheila Alexandra Belini Nishiyama PhD, Márcia Maria dos Anjos Szczerepa PhD, \\ Celso Luiz Cardoso PhD and Maria Cristina Bronharo Tognim PhD \\ Department of Basic Health Sciences, State University of Maringá, Maringá, Paraná, Brazil
}

To the Editor-The contamination of the environment and the hands of health professionals, transfer of patients, and movements of health professionals between hospitals are all possible routes for the dissemination of Acinetobacter baumannii. ${ }^{1-3}$ In our region during 2004-2008, an endemic carbapenem-resistant A. baumannii (CRAb) was detected. ${ }^{4}$ Later, it was verified (2011-2014), with a change in the dissemination mode of this microorganism (ie, the endemic situation to polyclonal dissemination). ${ }^{5}$ However, the routes of spread of $A$. baumannii have not yet been established.

In this study, we analyzed the effect of constructing a new ICU in a Brazilian hospital on the dissemination of A. baumannii. In the first 6 months, 22 clinical isolates were collected from an old ICU ( 12 beds), and in the next 6 months, 26 clinical isolates were collected from a newly installed ICU (24 beds).

In the new ICU, the presence of $A$. baumannii in the environment was investigated for a period of up to 15 days before and 15 days after patient admission to the unit. The samples were collected from bedside table, antiseptic dispenser, cardiac monitor, infusion pump, and bedrail, using sterile swabs moistened with sterile saline solution. Each swab was then used to inoculate a MacConkey agar plate.

The identification and antimicrobial susceptibility of bacterial isolates were assessed using a BD Phoenix system (Becton, Dickinson and Company, Franklin Lakes, NJ, USA). The minimum inhibitory concentrations of imipenem, meropenem, and polymyxin B were confirmed using the agar-dilution method. ${ }^{6}$

A multiplex PCR assay was performed to detect the presence of MBL genes ( $b l a_{\mathrm{IMP}}, b l a_{\mathrm{VIM}}, b l a_{\mathrm{GIM}}, b l a_{\mathrm{SPM}}$, and $\left.b l a_{\mathrm{SIM}}\right)$ and oxacillinase genes $\left(b l a_{\text {OXA23 }}, b l a_{\text {OXA24 }}, b l a_{\text {OXA51 }}\right.$, and $\left.b l a_{\text {OXA58 }}\right){ }^{7,8}$

Molecular typing was performed with enterobacterial repetitive intergenic consensus-polymerase chain reaction (ERIC-PCR) assays. Computer-assisted analysis was performed with BioNumerics version 6.5 software (Applied Maths, Sint-Martens-Latem, Belgium) with Dice correlation coefficient $\geq 0.93$. $^{9}$

Author for correspondence: Maria Cristina Bronharo Tognim, Laboratório de Microbiologia, Departamento de Ciências Básicas da Saúde, Universidade Estadual de Maringá, Avenida Colombo 5790, Maringá, Paraná, CEP 87020-900, Brasil. E-mail: mcbtognim@uem.br or cristinatognim@gmail.com

Cite this article: Moreira RRB et al. (2018) Dissemination of Acinetobacter baumannii OXA-23 in old and new intensive care units without transfer of colonized patients. Infection Control \& Hospital Epidemiology 2018, 39, 1135-1137. doi: 10.1017/ice.2018.168
In total, $48 \mathrm{~A}$. baumannii were isolated. The clinical and colonization isolates were obtained from tracheal aspirates $(n=26)$, urine $(n=6)$, blood $(n=5)$, cerebrospinal fluid $(n=1)$, wound secretion $(n=1)$, and nasal swabs $(n=4)$, oral swabs $(\mathrm{n}=4)$, and axillar swabs $(\mathrm{n}=1)$. During the study period, no patient infected or colonized with A. baumannii was transferred from the old to the new ICU, and no A. baumannii isolates were detected in the environment of the new ICU.

Of the 48 isolates, $65 \%$ and $50 \%$ were resistant to imipenem and meropenem, respectively. The most effective of the antibiotics tested was polymyxin B (100\% sensitivity), followed by tetracycline (73\%) and tobramycin (52\%). Comparing the isolates of A. baumannii from the 2 ICUs revealed an increase in resistance to imipenem in the isolates from the new unit (from 50\% to 69\%).

All isolates carried $b l a_{\mathrm{OXA} 51}$, and $29(60 \%)$ also carried $b l a_{\text {OXA23 }}$ (14 isolates from the old ICU and 15 isolates from the new unit). No strain was identified as a producer of MBL, OXA-58 or OXA-24.

The findings that $60 \%$ of the A. baumannii isolates showed the $b l a_{\text {OXA23 }}$ gene and that $11(38 \%)$ were susceptible to carbapenems can be explained. The expression of resistance depends not only on the presence of the $b l a_{\mathrm{OXA} 23}$ gene but also on its association with an insertion sequence, such as ISAbal, which enhances the expression of the $b a_{\text {OXA23 }}$ gene. These findings are worrisome because this insertion sequence may be inserted into a plasmid that has a high capacity for mobilization and dissemination. ${ }^{10}$

Molecular typing by ERIC-PCR of the $48 \mathrm{~A}$. baumannii isolates detected 17 different clusters (Fig. 1, A-Q). Two clusters were detected in both ICUs (I and J). Cluster J was detected in a patient admitted to the old ICU (negative for $b l_{a_{\mathrm{OXA} 23}}$ ) and in another patient admitted to the new ICU (positive for $b l a_{\text {OXA23 }}$ ). Cluster I was detected in 10 patients ( 5 in the old and 5 in the new ICU). This cluster was identical to the endemic CRAb detected in our region. ${ }^{5}$

The old ICU showed no distinct clusters, and the new ICU had 10 clusters. The most common clusters were $\mathrm{B}, \mathrm{I}$, and $\mathrm{H}$, which comprised $\sim 50 \%$ (23 of 48 ) of all A. baumannii isolates. Clusters B and $\mathrm{H}$ were isolated only in the new ICU, while cluster I was detected in both units. All clusters showed at least 1 isolate carrying $b l a_{\text {OXA23 }}$.

Molecular typing revealed a certain degree of clonal diversity, and despite the construction of the new ICU, several $A$. baumannii-producing OXA-23 coexist, making control more 